\title{
Research in High-Altitude and Mountain Emergency Medicine: Is Methodology Key?
}

\author{
Hermann Brugger, ${ }^{1}$ Matiram Pun, ${ }^{1}$ Erik R. Swenson, ${ }^{2}$ and Markus Falk ${ }^{1}$
}

$\mathbf{I}_{\mathrm{c}}^{\mathrm{s}}$ N 1878, The PIONEERING PHysiologist Paul Bert discovered that the oxygen partial pressure in the inspired air is responsible for altitude sickness, and not the barometric air pressure as previously suspected (Marotte, 2006). Bert used for the first time a decompression chamber and laid the foundation for 150 years of continuous research into highaltitude (HA) medicine and biology. Nonetheless, even to this day, the pathophysiological mechanisms of hypoxia maladaptation are not thoroughly explored, and the question why some humans get sick while others do not seems unanswered.

Methods of HA research have followed several parallel routes during this history. Most observations, experiments, and clinical trials were undertaken at altitude research stations such as the Observatoire Vallot (1890) on Mont Blanc, France, at $4358 \mathrm{~m}$, the Capanna Regina Margherita (1893) on Monte Rosa, Italy, at $4554 \mathrm{~m}$, the Barcroft Station (White Mountain Research Center) in California, United States, at $3800 \mathrm{~m}$ and the Pyramid Laboratory at Lobuje, Nepal (West et al., 2013) at $5050 \mathrm{~m}$. Another commonly used method to observe human adaptation to altitude was accompanying climbers on their HA expeditions, which began with the Silver Hut Expedition in 1963 lead by Sir Edmund Hillary (Milledge, 2010). Furthermore, normobaric or depressurized laboratories in North America and in Europe have been used to investigate HA physiology on humans under normobaric and hypobaric conditions. Although all these three settings seem similar in teasing out the effects of hypoxia, they tend to differ in terms of stimulus, strength, and responses. It should also be noted that studies with exposure to altitudes $>5000 \mathrm{~m}$ have continued occasionally (Grocott et al., 2010).

Emergency medicine in the mountains commenced decades later with the foundation of the International Commission of Mountain Emergency Medicine, ICAR MEDCOM, in 1948, although systematic research remained very sparse until the 1990s when the number of studies showed a substantial increase, especially on hypothermia and cold injuries.

In both fields, however, researchers have struggled with a series of methodological problems. The majority of field studies in HA and mountain emergency medicine work with small sample sizes typically in prospective studies (Strapazzon et al., 2011). The mechanistic physiological studies and invasive clinical studies tend to have small sample sizes owing to their complex methodology, difficulty in recruiting study volunteers, and high-financial burden. Populations are heterogeneous in many observational studies on trekkers and climbers, which renders the responses highly variable with respect to ethnicity, age, gender, and altitude of residence. The dropout rates in field studies tend to be high due to various factors such as illnesses, physical exertion, compliance, logistics, adverse weather, and changes in itinerary of trekking/expedition. The frequently used HA field study settings - White Mountain Research Station of California (Sea Level to $3800 \mathrm{~m}$ ), Capanna Regina Margherita in the Alps (490-4559 m), and Pheriche-Lobuje of Nepal (4200-5000 m) - clearly differ in altitudes and ascent rates. The hypoxic stimulus in these studies is different and results may not be readily comparable.

With small sample sizes $(n<30)$, only large differences or rates can be detected. Therefore, many aspects may remain hidden due to the low power (type II error or high false negative rate). The only possibilities to overcome this consist in reducing measurement error and by using more homogeneous samples (less variability between cases with respect to possible cofactors such as age, gender, or environmental factors). This, however, has a price. Less measurement error often dictates higher costs due to better equipment and training and more homogeneous samples suffer from less generalizability of results.

Moreover, in field studies we have almost no control over confounding environmental factors such as exposure to cold, wind, humidity, precipitation, or peer pressure in an organized trekking party. These factors, particularly, the possible interactions between mild or moderate hypothermia and hypoxia, have not been studied and have been widely neglected in most studies.

To summarize the sources of potential biases, the small sample size along with a high interindividual variability and a myriad of confounding environmental factors are the reasons

\footnotetext{
${ }^{1}$ Institute of Mountain Emergency Medicine, EURAC Research, Bolzano, Italy.

${ }^{2}$ VA Puget Sound Health Care System, University of Washington, Seattle Washington.

(C) Hermann Brugger et al., 2018; Published by Mary Ann Liebert, Inc. This Open Access article is distributed under the terms of the Creative Commons Attribution Noncommercial License (http://creativecommons.org/licenses/by-nc/4.0/) which permits any noncommercial use, distribution, and reproduction in any medium, provided the original author(s) and the source are cited.
} 
why the outcomes of clinical field studies in HA and mountain emergency medicine have varied widely and are thus difficult to compare.

One important step to standardize HA research was the development of acute mountain sickness (AMS) scoring systems (Environmental Symptom Questionnaire and Lake Louise Scoring System) based on the consensus of 35 experts from 14 different countries in 1991 and 1993 at the International Hypoxia Symposia held at Lake Louise, Canada (Hackett and Oelz, 1991; Roach et al., 1993). The AMS scoring systems have been extremely instrumental in improving our understanding of acute HA illnesses and the reporting of HA clinical results (Sampson et al., 1983; Roach et al., 1993).

In emergency medicine, the establishment of the Utstein Style, a uniform data reporting protocol, substantially contributed to improving the scientific evidence since 1991 (Cummins et al., 1991; Jacobs et al., 2004). Based on the consensus of an expert group, a set of data elements was defined, which nowadays is standard in the research of out-ofhospital cardiac arrest. These recommendations have enabled investigators to perform meta-analyses and compare studies.

Presently, reproducibility of scientific results ("reproducibility crisis") is considered the most urgent problem in science, as Nature recently stated (Baker, 2016). A survey revealed that $<50 \%$ of all medical study results can be reproduced, which is quite worrisome, because it can be suspected that this rate may be even higher in HA and mountain emergency medicine research due to the factors already mentioned. This was very recently highlighted in an initial report of successful prevention of AMS by inhaled budesonide, which could not be replicated in two well-planned and executed follow-on studies (Zheng et al., 2014; Lipman et al., 2017).

There is reason to believe that quality of HA research can be improved. In our mind, the STrengthening Altitude Research (STAR) project, proposed in this issue of the journal for more uniform reporting in HA clinical research (Brodmann et al., 2016), is expected to be an effective way of enhancing research quality.

Hypoxia exposure studies can be performed in normobaric and hypobaric chambers to reproduce and confirm results of field studies under controlled conditions, as a complementary option. In 2018, the so called terraXcube, a large environment simulator, will be functional in Bolzano, Italy, run by the Institute of Mountain Emergency Medicine at the EURAC research center (Brugger et al., 2016). This new infrastructure will simulate hypobaric hypoxia up to an altitude of $9000 \mathrm{~m}$, with temperatures between -40 and $+60^{\circ} \mathrm{C}$, and the addition of wind, rain, and snowfall. These factors can be driven separately or in combination, in contrast to their unpredictable and uncontrollable occurrence in the field.

In the future, both the STAR project and terraXcube facility are expected to have significant contribution in HA clinical research. Lastly, the International Society of Mountain Medicine as an umbrella organization will support the process of consensus finding, optimizing research methods to the highest possible level of quality, and disseminating the results.

\section{Acknowledgment}

The authors thank the Department of Innovation, Research and University of the Autonomous Province of Bozen/Bolzano for covering the Open Access publication costs.

\section{Author Disclosure Statement}

No competing financial interests exist.

\section{References}

Baker M. (2016). 1500 scientists lift the lid on reproducibility. Nature 533:452-454.

Brodmann M, Pun M, Strapazzon GS, Zafren K, Maggiorini M, Hackett PH, Bartsch P, Malacrida S, and Brugger $\mathrm{H}$. (2016). STrengthening Altitude Research (STAR): A Guideline for High Altitude Medicine and Research. In: In: $7^{\text {th }}$ World Congress of Mountain \& Wilderness Medicine A combined meeting of the International Society for Mountain Medicine and the Wilderness Medical Society July 30August 4, 2016 Telluride, Colorado. High Alt Med Biol 17:269.

Brugger H, Nollo A, Villardi A, Turner R, and Strapazzon GS. (2016). EES, Extreme Environment Simulator. In: $7^{\text {th }}$ World Congress of Mountain \& Wilderness Medicine. A combined meeting of the International Society for Mountain Medicine and the Wilderness Medical Society July 30-August 4, 2016 Telluride, Colorado. High Alt Med Biol 17:269-270.

Cummins RO, Chamberlain DA, Abramson NS, Allen M, Baskett PJ, Becker L, Bossaert L, Delooz HH, Dick WF, Eisenberg MS, et al. (1991) Recommended guidelines for uniform reporting of data from out-of-hospital cardiac arrest: The Utstein Style. A statement for health professionals from a task force of the American Heart Association, the European Resuscitation Council, the Heart and Stroke Foundation of Canada, and the Australian Resuscitation Council. Circulation 84:960-975.

Grocott MP, Martin DS, Wilson MH, Mitchell K, Dhillon S, Mythen MG, Montgomery HE, and Levett DZ. (2010). Caudwell xtreme Everest expedition. High Alt Med Biol 11:133-137.

Hackett PH, and Oelz O. (1991). The Lake Loiuse Consensus on the definition and quantification of altitude illness. In: Hypoxia and Mountain Medicine. JR Sutton, G Coates, CS Houston, eds. Pergamon Press, Oxford, UK. pp. 327-330.

Jacobs I, Nadkarni V, Bahr J, Berg RA, Billi JE, Bossaert L, Cassan P, Coovadia A, D'Este K, Finn J, et al. (2004). Cardiac arrest and cardiopulmonary resuscitation outcome reports: Update and simplification of the Utstein templates for resuscitation registries: A statement for healthcare professionals from a task force of the International Liaison Committee on Resuscitation (American Heart Association, European Resuscitation Council, Australian Resuscitation Council, New Zealand Resuscitation Council, Heart and Stroke Foundation of Canada, InterAmerican Heart Foundation, Resuscitation Councils of Southern Africa). Circulation 110:3385-3397.

Lipman GS, Pomeranz D, Burns P, Phillips C, Cheffers M, Evans K, Jurkiewicz C, Juul N, and Hackett P. (2017). Budesonide Versus Acetazolamide for Prevention of Acute Mountain Sickness. Am J Med. DOI:10.1016/j.amjmed .2017.05.034

Marotte H. (2006). The exposure of man to altitude when flying: From Paul Bert to today. J Soc Biol 200:251-255.

Milledge JS. (2010). The silver hut expedition, 1960-1961. High Alt Med Biol 11:93-101.

Roach RC, Bartsch P, Hacket PH, and Oelz O; Committee atLLASC. (1993). The Lake Louise Acute Mountain Sickness Scoring System. In: Hypoxia and Molecular Medicine. JR Sutton, CS Houston, G Coates, eds. Burlington, VT: Queen City Press, pp. 272-274. 
Sampson JB, Cymerman A, Burse RL, Maher JT, and Rock PB. (1983). Procedures for the measurement of acute mountain sickness. Aviat Space Environ Med 54:1063-1073.

Strapazzon G, Procter E, and Brugger H. (2011). Quest for evidence based practice in mountain emergency medicine. High Alt Med Biol 12:399-400.

West JB, Schoene RB, Luks AM, and Milledge JS. (2013). High Altitude Medicine and Physiology. CRC Press, Taylor \& Francis Group, Milton Park, Abingdon, UK.

Zheng CR, Chen GZ, Yu J, Qin J, Song P, Bian SZ, Xu BD, Tang XG, Huang YT, Liang X, et al. (2014). Inhaled budesonide and oral dexamethasone prevent acute mountain sickness. Am J Med 127:1001-1009.e2.
Address correspondence to: Hermann Brugger, MD

Institute of Mountain Emergency Medicine EURAC Research Viale Druso 1 Bolzano 39100 Italy

E-mail: hermann.brugger@eurac.edu

Received September 19, 2017; accepted in final form September 21, 2017. 